Methods: Synovial fluid mononuclear cells (SFMCs), fibroblast like synovial cells (FLSs) and peripheral blood mononuclear cells (PBMCs) were obtained from a study population consisting of patients with active RA or peripheral SpA with at least one swollen joint (for obtaining synovial fluid) $(n=14)$. SFMCs were cultured for 48 hours with and without addition of a MK2 inhibitor (Celgene) at $1000 \mathrm{nM}, 333 \mathrm{nM}$ and $111 \mathrm{nM}$ and supernatants were analysed by the Olink proseek multiplex interferon panel and commercially available ELISA assays. Because FLSs are only found in small amounts among SFMCs, autologous co-cultures of FLS and PBMCs and SFMCs were also used. SFMCs cultured for 21 days were used to study inflammatory macrophage differentiation and osteoclastogenesis.

Results: In SFMCs cultured for 48 hours, the MK2 inhibitor decreased the production of CXCL9 $(p<0.001)$, CXCL10 $(p<0.01)$, HGF $(p<0.01), C X C L 11$ $(p<0.01)$, TWEAK $(p<0.05)$, and IL-12B $(p<0.05)$ and increased the production of CXCL5 $(p<0.0001)$, CXCL1 $(p<0.0001)$, CXCL6 $(p<0.001)$, TGF $\alpha(p=0.01)$, MCP-3 $(p<0.01)$, LAP TGF $\beta(p<0.05)$ dose-dependently after Bonferroni correction (all corrected $P$ values). At the highest concentration, the MK2 inhibitor also decreased MCP-1 production $(p<0.05)$. In FLS-SFMC co-cultures, the MK2 inhibitor decreased MCP-1 production $(p<0.05)$ but did not change the production of DKK1 and MMP3. In FLS-PBMC co-cultures, the MK2 inhibitor decreased the production of MCP-1 $(p<0.0001)$, increased MMP3 production $(p<0.05)$ but did not change DKK1 production. In SFMCs cultured for 21 days as a model of inflammatory macrophage differentiation and osteoclastogenesis, the MK2 inhibitor decreased the production of MCP-1 $(p<0.05)$ and tartrate-resistant acid phosphatase (TRAP) $(p<0.05)$ but did not change the production of IL-10.

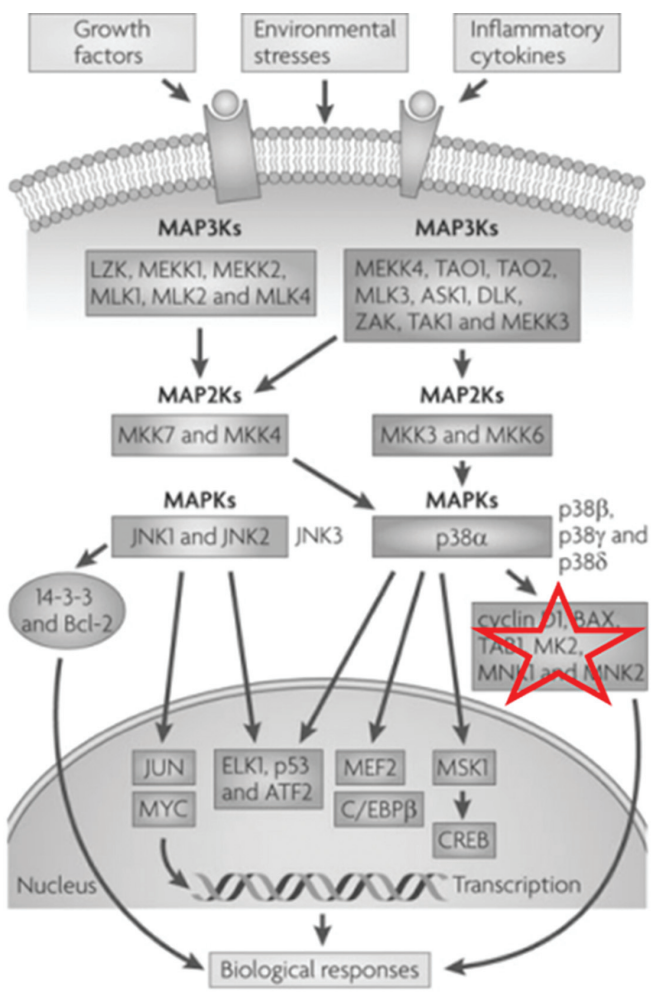

Abstract THU0096 - Figure 1. Modified from Wagner \& Nebreda, Nature Review Cancer, 2009.

Conclusions: This study reveals the effects of a MK2 inhibitor in ex vivo models of immune mediated inflammatory arthritis. The MK2 inhibitor changed the secretory profile of SFMCs and decreased inflammatory osteoclastogenesis. Taken together, this points to a role of this MK2 inhibitor in attenuating inflammatory and destructive arthritis.

Disclosure of Interest: T. Kragstrup: None declared, A. Mellemkjær: None declared, M. Nielsen: None declared, L. Heftdal: None declared, P. Schafer Shareholder of: Celgene, Employee of: Celgene, B. Deleuran: None declared DOI: 10.1136/annrheumdis-2018-eular.5747

\section{THU0097 PHOSPHODIESTERASES 4 (PDE4) INHIBITOR AMELIORATES EXPERIMENTAL ARTHRITIS}

W. Chen ${ }^{1}$, Z. Xu ${ }^{2}$, H. Wee ${ }^{3}$, G.S. Lewis ${ }^{3}$, N. Olsen ${ }^{2}$, J. Lin ${ }^{1}$, S.G. Zheng ${ }^{2}{ }^{1}$ the first affiliated hospital, college of medicine, zhejiang university, Hangzhou, China; ${ }^{2}$ Division of Rheumatology, Department of Medicine, Penn State University Hershey College of Medicine Hershey; ${ }^{3}$ Department of Orthopaedics and Rehabilitation, Penn State University Hershey College of Medicine Hershey, Hershey, USA

Background: Rheumatoid arthritis (RA) is a chronic inflammatory bone-destructive disorder with autoimmune features. Apremilast is a novel phosphodiesterases 4 (PDE4) inhibitor suppressing immune and inflammatory responses.

Objectives: We assessed the anti-inflammatory and bone protection effects of apremilast in collagen Cll induced arthritis (CIA)models.

Methods: Apremilast was given starting from day 14 after immunisation, we investigated whether apremilast $(5 \mathrm{mg} / \mathrm{kg}$ or $25 \mathrm{mg} / \mathrm{kg}$ ) can ameliorate arthritis onset. Bone erosion was measured by histological and micro-computed tomographic analysis. Anti-mouse type II collagen (CII) antibody levels were measured by enzyme-linked immunosorbent assay. Human cartilage and rheumatoid arthritis (RA) synovial fibroblasts (RASFs) implantation in the severe combined immunodeficiency (SCID) mouse model of RA were used to study the role of apremilast in suppression of RASFs destroying cartilage in vivo.

Results: We found that apremilast therapy delayed arthritis onset and reduced arthritis scores in CIA model at a different dose, compared to CIA model and blank vector (figure 1A). Total serum IgG, IgG1, IgG2a, and lgG2b were all decreased in apremilast groups. Furthermore, apremilast can prevent $\mathrm{ClA}$ mice from bone erosion by CT analysis. High dose of apremilast $(25 \mathrm{mg} / \mathrm{kg}$ ) was superior to low dose $(5 \mathrm{mg} / \mathrm{kg}$ ) in treating CIA (figure 1B, C). Apremilast treatment can inhibit destroy and migratory ability of RASFs to cartilages. Compared to the model group, Apremilast treatment significantly reduced the invasion scores in both primary implant and contralateral implant.

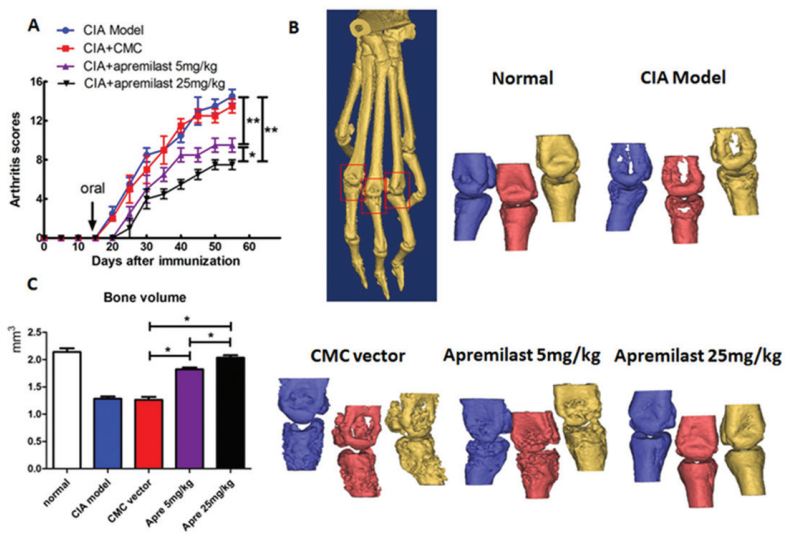

Conclusions: Our data suggest that apremilast was effective in preventing arthritis and bone erosion in CIA model, implicating a potential promise of therapy on rheumatoid arthritis.

Acknowledgements: This work is supported in part by the grants from the Celgene Foundation, NIH R01 059103, National Natural Science Foundation of China (81671611, 81701600).

Disclosure of Interest: None declared

DOI: 10.1136/annrheumdis-2018-eular.4989

\begin{tabular}{l|l}
\hline THU0098 & COMBINATION THERAPY OF RAPAMYCIN AND A \\
GLUTAMINE ANTAGONIST FACILITATES THE \\
EXPANSION OF MYELOID-DERIVED SUPPRESSOR \\
CELLS AND AMELIORATES ARTHRITIS IN SKG MICE
\end{tabular}

Y. Ueda, J. Saegusa, T. Okano, S. Sendo, H. Yamada, Y. Ichise, I. Naka, S. Takahashi, K. Akashi, A. Onishi, A. Morinobu. Rheumatology and Clinical immunology, Kobe University Graduate School of Medicine, Kobe, Japan

Background: Myeloid-derived suppressor cells (MDSCs) are a heterogeneous population of immature cells that increase in the pathological state such as tumour or inflammation and have the immunosuppressive ability. MDSCs have been 
reported to ameliorate arthritis in several mice models. Mechanistic target of rapamycin (mTOR) pathway and glutaminolysis activate cooperatively in the differentiation from myeloid progenitors to mature myeloid cells such as dendritic cells, macrophages, or osteoclasts as well as the activation of effector T cells and the differentiation of Th1 and Th17 cells. Although rapamycin has reported to facilitate the expansion of MDSCs and their immunosuppressive ability, the effect of the inhibitor of glutaminolysis on MDSCs is still unknown.

Objectives: The aim of this study is to evaluate the facilitative effects of the inhibition of mTOR pathway and glutaminolysis on MDSCs in a mouse model of rheumatoid arthritis.

Methods: Bone marrow (BM) cells from untreated Balb/c mice were cultured for 5 days under granulocyte-macrophage colony-stimulating factor (GM-CSF) stimulation with four patterns of drugs; 1) DMSO (control), 2) rapamycin (Rapa), 3) 6Diazo-5-oxo-I-norleucine (DON; a glutamine antagonist), or 4) the combination of rapamycin and DON (Rapa $+\mathrm{DON})$. Cultured BM cells were analysed by flow cytometry. Cultured MDSCs were isolated by manual MACS and analysed their immunosuppressive characters by co-culture with CFSE-dyed $\mathrm{CD} 4^{+} \mathrm{T}$ cells. Rapa or Rapa +DON were administered intraperitoneally to arthritic SKG mice induced by Zymosan A injection.

Results: We found that DON suppressed the differentiation of dendritic cells (DC) in a dose-dependent manner and the addition of Rapa on DON inhibited the differentiation of macrophages in vitro. The proportions of the phenotype of MDSCs were increased with administrations of Rapa or DON, and large part of them were $\mathrm{Ly}_{6 \mathrm{G}}{ }^{+}$cells (the phenotype of polymorphonuclear MDSCs; PMN-MDSCs). Rapa \pm DON significantly increased the expressions of TGF- $\beta$ and PD-L1 and the inhibitory capacity of Ly6G+ ${ }^{+}$PMN-MDSCs. Rapa +DON significantly suppressed arthritis more efficiently in SKG mice than Rapa in vivo. (see figure 1)

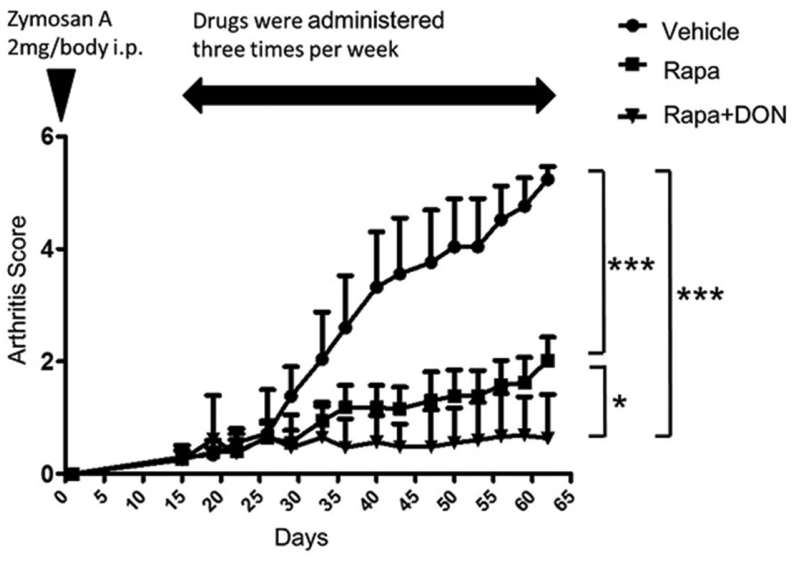

Conclusions: The combination of rapamycin and a glutamine antagonist facilitates the expansion of PMN-MDSCs in vitro and ameliorates arthritis in SKG mice in vivo.

Disclosure of Interest: None declared

DOI: 10.1136/annrheumdis-2018-eular.3598

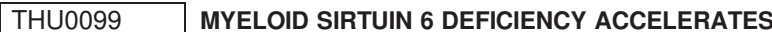 EXPERIMENTAL AND HUMAN ARTHRITIS BY INCREASING MACROPHAGE INFILTRATION INTO SYNOVIUM}

Y.S. Suh ${ }^{1}$, Y.-H. Cheon ${ }^{2}$, M.Y. Kim², S.J. Woo ${ }^{3}$, H.S. Noh², B.-H. Park ${ }^{3}$, S.-I. Lee ${ }^{2}$ ${ }^{1}$ Gyeongsang National University Changwon Hospital, Changwon; ${ }^{2}$ Gyeongsang National University Hospital, Jin-Ju; ${ }^{3}$ Chonbuk National University Medical School, Jeonju, Korea, Republic of Ireland

Background: Rheumatoid arthritis (RA) is an autoimmune inflammatory disease of the joints and is characterised by immune cell infiltration, synovial hyperplasia, and destruction of cartilage and underlying bone ${ }^{1}$. Myeloid derived monocytes and macrophages secrete a variety of cytokines such as tumour necrosis factor- $\alpha$ (TNF- $\alpha$ ), interleukin (IL) $-1 \beta$ and IL-6, all of which perpetuate and amplify the vicious cycle of chronic inflammatory pathways ${ }^{2}$. Indeed, macrophage numbers in the synovium and serum levels of monocyte-derived cytokines correlate well with clinical symptoms and degree of joint damage in $\mathrm{RA}^{3}$

Objectives: We recently reported that myeloid sirtuin 6 (Sirt6) is a critical determinant of phenotypic switching and the migratory responses of macrophages ${ }^{4}$.
Given the prominent role of macrophages in rheumatoid arthritis (RA) pathogenesis ${ }^{5}$, we tested whether myeloid Sirt6 deficiency affects the development and exacerbation of RA

Methods: Arthritis was induced in wild type and myeloid Sirt6 KO (mS6KO) mice using collagen-induced and $\mathrm{K} / \mathrm{BxN}$ serum transfer models. Peripheral blood mononuclear cells (PBMC) and synovial fluid macrophages were obtained from patients with RA and osteoarthritis and used for comparisons of Sirt6 expression and inflammatory activities.

Results: Based on clinical scores, ankle thickness, pathology and radiology, arthritis was more severe in mS6KO mice relative to wild type with a greater accu mulation of macrophages in the synovium. Consistently, myeloid Sirt6 deficiency increased the migration potential of macrophages toward synoviocytes-derived chemoattractants. Mechanistically, Sirt6 deacetylates forkhead box protein $\mathrm{O} 1$ to trigger its nuclear export and proteasomal degradation. Lastly, PBMC and macrophages isolated from RA patients exhibited lower Sirt6 expression compared with those from osteoarthritis patients or healthy subjects and their Sirt6 activity was inversely correlated with disease severity of the patients.

Conclusions: Our data identify a role of myeloid Sirt6 in clinical and experimental RA and suggest that myeloid Sirt6 may be an intriguing therapeutic target.

\section{REFERENCES:}

[1] Ospelt C. Synovial fibroblasts in 2017. RMD Open 2017;3(2):e000471.

[2] Gierut A, Perlman H, Pope RM. Innate immunity and rheumatoid arthritis. Rheum Dis Clin North Am 2010;36(2):271-96.

[3] Kinne RW, S tuhlmuller B, Burmester GR. Cells of the synovium in rheumatoid arthritis. Macrophages. Arthritis Res Ther 2007;9(6):224

[4] Lee $\mathrm{Y}, \mathrm{Ka}$ SO, Cha HN, et al. Myeloid sirtuin 6 deficiency causes insulin resistance in high-fat diet-fed mice by eliciting macrophage polarization toward an M1 phenotype. Diabetes 2017;66(10):2659-68.

[5] Lee HS, Ka SO, Lee SM, et al. Overexpression of sirtuin 6 suppresses inflammatory responses and bone destruction in mice with collageninduced arthritis. Arthritis Rheum 2013;65(7):1776-85.

Disclosure of Interest: None declared

DOI: 10.1136/annrheumdis-2018-eular.4507

\section{THURSDAY, 14 JUNE 2018}

\section{Rheumatoid arthritis - prognosis, predictors and outcome}

\section{THU0100 WORK IMPAIRMENT IN RHEUMATOID ARTHRITIS IS HIGHLY CORRELATED WITH DEPRESSION}

A.D. Houssien, J. Galloway. Academic Rheumatology department, King's College London, London, UK

Background: Work outcomes are highly relevant in rheumatoid arthritis. Almost a third of people are no longer in employment by 2 years after diagnosis. Remain ing in the workforce has positive attributes in terms of health outcome and quality of life.

Objectives: We set out to study the relationship between work and depression in rheumatoid arthritis.

Methods: We used routinely collected data from a hospital rheumatoid arthritis cohort that has systematically collected longitudinal data on disease measures, mental health, and work impairment. The Work and Social Adjustment Scale was used to estimate work impact, and we used question 2 (scale 0-8, above 6 indicating severe impairment) for analyses. Mood was assessed using the PHQ2. Cross sectional analyses were used to describe the relationship between work impairment and depression, adjusting for disease severity factors. We then analysed the temporal relationship between work impairment and mood.

Results: In total 283 patients had data available for analysis. Mean age was 53 years (SD 15), 81\% were female, median disease duration 6 years (IQR 0 to 10) $74 \%$ were seropositive (RF or CCP), mean baseline DAS28 score 3.8 (SD 1.7) baseline HAQ 1.2 (SD 0.9). At baseline 69\% reported no depression, $13 \%$ reported some symptoms, $18 \%$ had probable major depression. $72 \%$ of patients reported some degree of work impairment, $14 \%$ reported severe impairment. There was a strong relationship between baseline depressive symptoms and work impairment: coefficient $2.4,95 \% \mathrm{Cl} 1.7$ to $3.0, \mathrm{p}<0.001$. Adjusting for age, gender, DAS28, HAQ: $1.3,95 \% \mathrm{Cl} 0.5$ to $2.0, \mathrm{p}=0.004$. Over time work impairment increased (linear regression coefficient: 0.2 per year $95 \% \mathrm{Cl} 0.1$ to 0.4 ), see figure 1. People with baseline low mood had an accelerated worsening of work impairment. 